\title{
Arbitrary real-time three-dimensional corporal object sensing and reconstruction scheme
}

\author{
R. S. Bhamber, ${ }^{2}$ T. Allsop, ${ }^{1, *}$ G. Lloyd, ${ }^{3}$ D. J. Webb, ${ }^{1}$ and J. D. Ania-Castanon ${ }^{2}$ \\ ${ }^{1}$ Aston Institute of Photonic Technologies, Department of Electronic Engineering, Aston University, Birmingham B4 $7 E T$, UK \\ ${ }^{2}$ Instituto de Óptica "Daza de Valdés”, (C.S.I.C.), C/ Serrano, 121, 28006 Madrid, Spain \\ ${ }^{3}$ Moog Insensys Ltd, Ocean House, Whittle Avenue, Segensworth West, Fareham PO15 5SX, UK \\ *Corresponding author: t.d.p.allsop@aston.ac.uk
}

Received May 9, 2012; revised July 16, 2012; accepted July 16, 2012;

posted July 17, 2012 (Doc. ID 167636); published August 22, 2012

\begin{abstract}
A real-time three-dimensional (3D) object sensing and reconstruction scheme is presented that can be applied on any arbitrary corporeal shape. Operation is demonstrated on several calibrated objects. The system uses curvature sensors based upon in-line fiber Bragg gratings encapsulated in a low-temperature curing synthetic silicone. New methods to quantitatively evaluate the performance of a 3D object-sensing scheme are developed and appraised. It is shown that the sensing scheme yields a volumetric error of $1 \%$ to $9 \%$, depending on the object. @ 2012 Optical Society of America

OCIS codes: $\quad 060.2370,060.3735,120.4820,110.6880,280.4788$.
\end{abstract}

One of the main goals driving forward the research and development of fiber-optic sensing systems is their application in the field of structural health monitoring $[\underline{1}, \underline{2}]$ and shape sensing $[3,4]$. There are several applications for shape-sensing, such as spatial awareness and control of robots in hazardous conditions (e.g., deep-water semisubmersible drilling platform and subsea production systems) $[\underline{5}, \underline{6}]$ or profiling minimally invasive (keyhole) surgery [7]], to name a few.

There are a large number of shape sensing systems that have fiber Bragg gratings (FBG) as the sensing elements that monitor the strain experienced by the sensors that are adhered to the object and use a shape determination algorithm based upon a strain mapping technique [1, $\underline{3}]$. Other sensing systems include fiber-optic loop sensors based on bend-induced loss; this technique is used in the commercially available system called ShapeTape [2] with others based upon distributed sensing, such as intrinsic Rayleigh backscattering employing optical frequency domain reflectometry [8]. Alternative approaches to shape sensing include employing camera systems using complex, shape-sensing recognition algorithms [9].

The three-dimensional (3D) shape-sensing system demonstrated in this Letter is based upon fiber bidirectional sensing elements that have the ability to distinguish both positive and negative curvature variation upon a twodimensional (2D) plane. The element consists of two FBGs that are temperature self-compensating due to the fact that the difference in wavelength is measured and calibrated to curvature (concave and convex) (see Fig. 1). The curvature information deduced from all sensing nodes within the scheme is processed by bespoke algorithms to construct a 3D representation of the object in question. As we will show, our method offers advantages in comparison to previously existing approaches, the most important of which is the real-time 3D shape reconstruction through tailor-made algorithms. The volumetric error of the sensing system was found for shapes with known volumes (i.e., arcs, ellipsoidal cylinders, and spheres) and ranged from $1 \%$ to $9 \%$ depending upon the shape being used. In addition, we present an efficient and, to the extend of our knowledge, entirely new approach to the evaluation of the performance of a 3D object shape sensing system, obtaining a figure-of-merit that should be a useful tool for researchers to compare shape reconstruction efficiencies using different measurement approaches.

Our sample sensing scheme possesses 20 sensing locations within the sensor array, each spatial location consisting of two FBGs spatially arranged one above the other separated by a small distance, typically $\sim 0.5 \mathrm{~mm}$ either side of the neutral axis to ensure the sensors respond in opposite fashions to curvature; see Figs. 1 and 2. All the FBGs are inscribed in series along a single fiber optic line (Fibrecore SM1500) separated by $1 \mathrm{~m}$ in the fiber (due to the limitations of the interrogation scheme employed). The overall array's shape was chosen specifically for the respiratory function monitoring of humans. The complete sensing array is encapsulated with a low temperature $\left(90^{\circ} \mathrm{C}\right)$ curing silicone rubber. Also this silicone rubber reduces the effect of transient ambient temperature changes on the FBGs, a schematic is shown in Fig. 1. Each FBG is approximately $7 \mathrm{~mm}$ long having a peak reflectivity of $5 \%$ along with a spectral bandwidth, FWHM, of $70 \mathrm{pm}$; all have approximately the same peak wavelength of $1550 \mathrm{~nm}$.

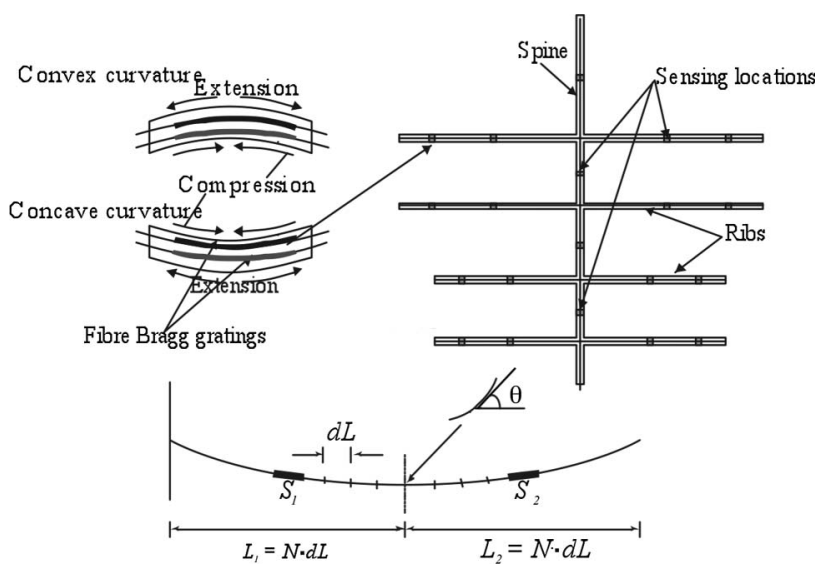

Fig. 1. Schematic of the curvature sensing array. 
These FBGs are specifically designed for the interrogation scheme employed for the sensing array, which is a based upon time division multiplexing (Moog Insensys Ltd.) [10]. The interrogator addresses sensors at a frequency of $500 \mathrm{~Hz}$; this sensing array consists of $42 \mathrm{de}-$ vices and thus the complete array is addressed 12 times per second.

A calibration of the curvature sensing nodes was first performed by bending of the silicone where the two FBG elements are located. A relationship between wavelength shift in nanometer and curvature experienced by each sensing node is established with typical average spectral sensitivities to curvature of $\Delta \lambda / \Delta R=0.80 \pm 0.12 \mathrm{~nm}$ with an overall curvature resolution of $0.125 \mathrm{~m}^{-1}$ determined by a combination of the minimum detectable wavelength shift and the inaccuracy in calibration procedure.

The generation of the shape from the sensing array formed from the curvature sensors is achieved by using the following algorithms. The distances between adjacent sensing nodes is known, along with the total lengths of fiber between sensing elements in the array; thus the arc lengths for each sensing location are known. Consider a single rib, one horizontal arm, which consists of four sensors; this is divided into four sections with each section consisting of $N$ segments, the last section being used to calculate the angle to the spine of the array to ensure a continuous line/curve between adjacent sensing sections, see Fig. 1. By combining all of the curvature values, coupled with the known spatial dimensions, we are able to generate the corresponding $x, y$ and $z$ coordinates of the sensor array. We can then reconstruct the entire sensing array in real-time in 3D space with a positional accuracy determined in the curvature calibration process that is translated into Euclidean space. With the sensing array reconstructed in 3D, see Figs. 2 and 3, we are now able to calculate the volume of the various sections of the system. The procedure consists of first generating a $2 \mathrm{D}$ mesh across the surface of the ribs, dynamically, by interpolating the points in between the sensor locations, both vertically and horizontally. Using this data, we are able to then select a region of interest and perform a 2D surface integration over the section of the mesh, thus obtaining a temporally dependent volume at any location of the array.

The system is evaluated by using known shapes and volumes and determining the error in the estimated

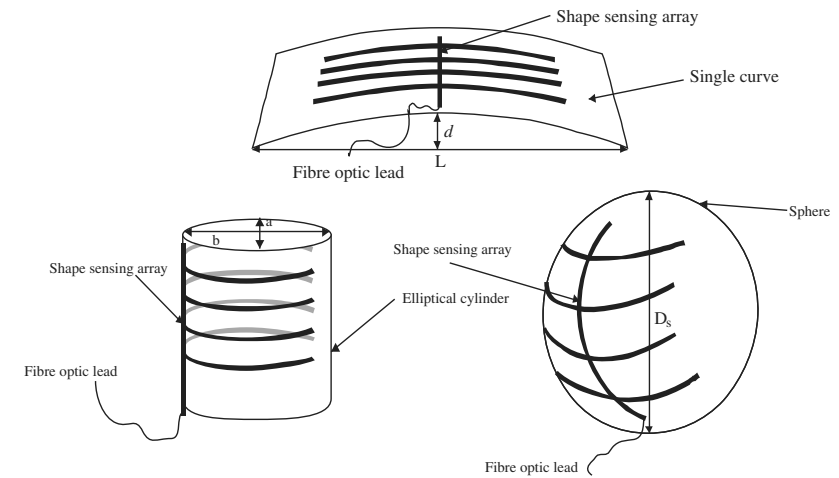

Fig. 2. Schematics of the shapes used to evaluate the shaping sensing scheme.

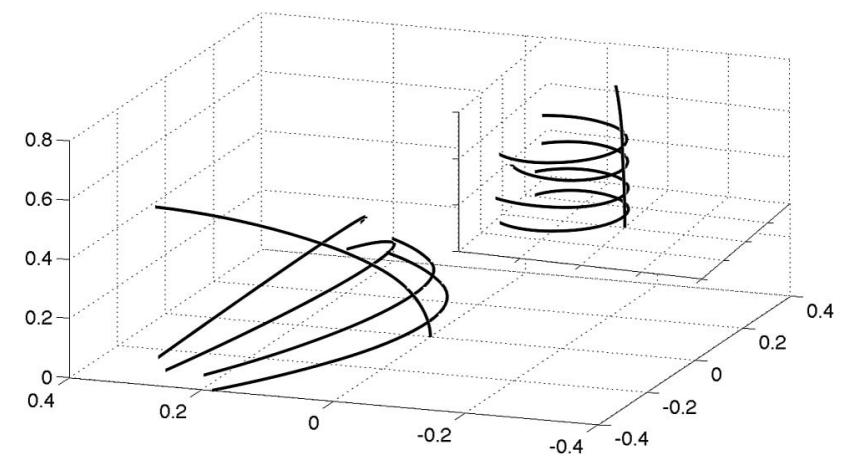

Fig. 3. 3D sensing array representation obtained from the algorithm, all axis units are meters.

volume of the shape. The shapes have been chosen to evaluate the system with increasing dimensional difficulty; the first shape is a simple curvilinear plane (set to various constant curvatures) which presents a single dimensional degree of freedom, second, an elliptical cylinder where the major and minor axes are varied, is a second degree of freedom; two-dimensions, and third, varying diameters of known spheres again increase to three degrees of freedom, see Fig. 2 .

The algorithm reproduces the shape of the object in contact with the shape sensing array. The volume error for the algorithm was calculated for the known shapes and volumes. The estimated volumetric error for the elliptic cylinders used was approximately $7.8 \%$ and for the spheres the error ranged from $1 \%$ to $9 \%$ depending upon the size.

To further evaluate the performance of the shapesensing system, new quantitative approaches are developed to obtain a figure of merit for any 3D arbitrary shape. Established methods for pattern recognition, such as cross correlation (the global shape matching and local shape matching) [11] and common shape-matching methods including shape distribution [12], shape context [13], and curvature scale space $[\underline{8}, \underline{14}]$ are generally limited to $2 \mathrm{D}$ or do not consider surface quality.

In order to quantitatively analyze any arbitrary $3 \mathrm{D}$ object, two procedures are devised, first the use of normalized cross-correlation functions for each dimension $(x, y, z)$, defined as

$$
f\left(S_{S} S_{T}\right)_{x, y, z}=\frac{1}{n} \frac{\sum_{x, y, z}\left(S_{x, y, z}-\langle S\rangle_{x, y, z}\right)\left(T_{x, y, z}-\langle T\rangle_{x, y, z}\right)}{\sigma(x, y, z) \sigma(x, y, z)},
$$

where $S_{S}$ is the reconstructed shape from the measurement, $S_{T}$ is the known 3D shape to be correlated, $S_{x, y, z}$ are the values of the shape in a given dimension $(x, y, z)$,

Table 1. Cross Correlation For Various Sphere Radii

\begin{tabular}{lccc}
\hline & \multicolumn{3}{c}{ Dimension } \\
\cline { 2 - 4 } Sphere Radius (m) & $X$ & $Y$ & $Z$ \\
\hline 0.29 & 0.9955 & 0.9572 & 0.9511 \\
0.315 & 0.9971 & 0.9555 & 0.9792 \\
0.36 & 0.9986 & 0.9837 & 0.9781 \\
\hline
\end{tabular}




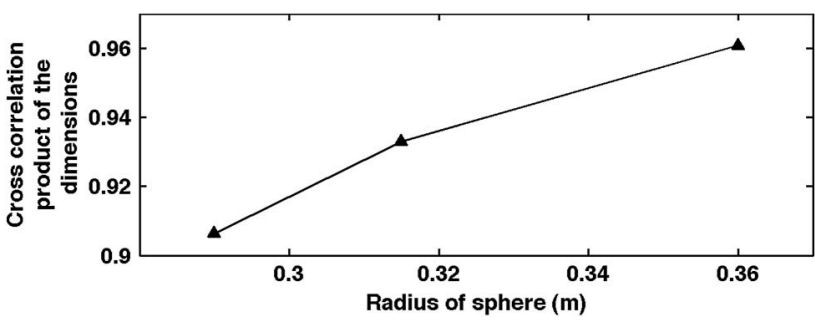

Fig. 4. The overall product of cross-correlation of the different spheres.

$T_{x, y, z}$ are the corresponding idealized values, \langle\rangle represents the average value in the given dimension and $\sigma$ is the standard deviation for a given dimension. Using this approach yields values between -1 to +1 for each dimension and the product of the $x, y, z$ components measure the validity of the reconstructed shape with regard to the reference object. Results are shown in Table 1 and Fig. 4 .

The second approach using a normalized vector innerproduct that consist of calculating the product for each preset $n$ points on the surface of the reconstructed shape $S_{S}$ and the known idealized shape $S_{T}$, this procedure is defined in the following equation:

$$
F\left(S_{S} S_{T}\right)_{n}=\frac{1}{n} \sum_{n} \frac{S_{S} \cdot S_{T}}{\left|S_{T}\right|^{2}} .
$$

This second procedure yields a numerical value describing the quality of the reconstructed shape's topology compared to the known topology of the reference object, for example for a perfect reconstruction, this procedure would produce one. For values greater or lower than one, results in an over or under estimation, respectively, of the physical topology of the reference object see Fig. $\underline{5}$. Relevant sources of error include imperfections in the alignment of the FBGs, as well as the assumption that the arms remain perpendicular to the spine and all wavelength shifts are due to bending.

Upon inspecting Table 1 we find all the cross-correlation values are above 0.95 , thus indicating good agreement between the known spheres and the measurements taken with the sensing scheme. Again this is verified by observing that the overall cross-correlation values, as seen in Fig. 4, are in excess of 0.9 , and the average inner-product approach is within $3 \%$ of the idealized case (which is 1 ). See Fig. 5. Again these results indicate that the sensing

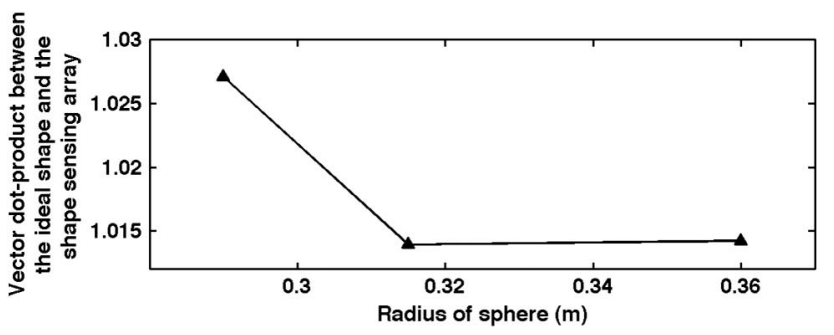

Fig. 5. The overall average scalar/dot product between the idealized shape and the shape reconstructed from the sensing array for the different spheres.

schemes reconstruction is an accurate representation of the original object to which the array is adhered to.

\section{References}

1. M. A. Davis, A. D. Kersey, J. Sirkis, and E. J. Friebele, J. Smart Mater. Struc. 5, 759 (1996).

2. L. Danisch, K. Englehart, and A. Trivett, Sens. Rev. 19, 106 (1999).

3. L. Yuan, J. Yang, C. Guan, Q. Dai, and F. Tian, Opt. Lett. 33, 578 (2008).

4. W. Yin, T. Fua, J. Liu, and J. Leng, Proc. SPIE 7292, 72921H (2009).

5. R. L. Hansen and W. P. Rickey, J. Petrol. Technol. 47, 675 (1995).

6. J. Pretlove, C. Skourup, and J. Gunnar, in SPE Intelligent Energy Conference and Exhibition (Society of Petroleum Engineers, Utrecht, The Netherlands, 2010), p. 128232-MS.

7. M. Eltaib and J. R. Hewit, Mechatronics 13, 1163 (2003).

8. R. G. Duncan, M. E. Froggatt, S. T. Kreger, R. J. Seeley, D. K. Gifford, A. K. Sang, and M. S. Wolfe, Proc. SPIE 6530, 65301S (2007).

9. J. M. Croom, D. C. Rucker, J. M. Romano, and R. J. Webster, in 2010 IEEE International Conference on Robotics and Automation (ICRA) (IEEE, 2010), pp. 4591-4596.

10. G. D. Lloyd, L. A. Everall, K. Sugden, and I. Bennion, IEEE Photon. Technol. Lett. 16, 2323 (2004).

11. L. Lv, Y. Liu, W. Zhang, C. Jiang, X. Chen, T. Chang, and M. Yuen, J. Softw. 4, 413 (2009).

12. C. Y. Ip, D. Lapadat, L. Sieger, and W. C. Regli, in Proceedings of the 7th ACM Symposium on Solid Modeling and Applications (SMA, Saarbrućken, Germany, 2002), pp. 273-280.

13. S. Belongie, J. Malik, and J. Puzicha, IEEE Trans. Pattern Anal. Mach. Intell. 24, 509 (2002).

14. F. Mokhtarian and R. Suomela, in Proceedings of the International Conference on Image Processing and its Applications, Seventh International Conference on (Conf. Publ. No. 465) (Manchester, UK, 1999), pp. 206-210. 